\title{
Three-dimensional vibration analysis of a cantilevered parallelepiped: Exact and approximate solutions
}

\author{
C. W. Lim \\ Department of Mechanical Engineering, The University of Hong Kong, Pokfulam Road, Hong Kong
}

(Received 6 April 1999; accepted for publication 20 August 1999)

\begin{abstract}
The paper investigates the hypothetical assumption of neglecting transverse normal stress in vibration analysis for cantilevered thick plates and rectangular parallelepiped. The analysis solves the three-dimensional elasticity energy functional including, as well as excluding, transverse normal stress and obtains free vibration solutions for a cantilevered parallelepiped. Although it is widely accepted, the omission of transverse normal stress is well justified in Kirchhoff-Love thin-plate theory and higher-order thick-plate models; the transverse normal stress effects and thickness extent to which the thick-plate models apply as the thickness increases are practically unknown. The inconsistency of assuming constant transverse normal displacement through thickness for thick-plate models is also addressed. The paper concludes that for a rectangular parallelepiped with thickness exceeding a certain limit, there is considerable discrepancy if transverse normal stress is neglected. (C) 1999 Acoustical Society of America. [S0001-4966(99)02312-7]
\end{abstract}

PACS numbers: 43.40.Dx [CBB]

\section{INTRODUCTION}

For decades, the analysis of thick plates has neglected the effects of transverse normal stress. The ReissnerMindlin first-order plate theory (Reissner, 1945; Mindlin, 1951) extended the classical Kirchhoff-Love thin-plate theory to analyze plates with considerable thickness. Transverse shear deformation was considered by including transverse shear strain effects in the analysis. The first-order theory assumes constant transverse shear strains through the plate thickness and renders a paradoxical implication that the transverse shear strain components do not vanish on the top and bottom surfaces. A shear correction factor $\left(\kappa=\pi^{2} / 12\right)$ was therefore derived by Reissner (1945) to account for this deficiency. Using this first-order theory, accurate vibration formulation and solutions have been reported for laminated curved beams (Qatu, 1993) and plates (Bert and Chen, 1978).

The inadequacy of the first-order shear deformation theory to overcome the nonvanishing shear strain effects stimulated the development of research in thick plates with various formulations of higher-order theories. One of the earliest attempts was initiated by Soler (1968), who expressed all dependent variables including displacement and stress components in Legendre polynomials. Other developments in the higher-order plate theory include Whitney and Sun (1973) with quadratic and linear distributions for in-plane and transverse displacements; Whitney and Sun (1974) with linear and quadratic distributions for in-plane and transverse displacements; and Iyengar et al. (1974) and Lo et al. (1977a,b) with cubic and quadratic distributions for in-plane and transverse displacements. Washizu (1980) expressed the in-plane displacement in a power series of transverse coordinate $(z)$ and simplified the function to a first-order expression. By imposing zero transverse shear-stress conditions at the free surfaces, Levinson (1980) developed a third-order plate theory with cubic in-plane displacement and constant transverse displacement without the requirement of a shear correction factor. Perhaps the most remarkable work on the third-order shear deformation theory for thick plates was attributed to Reddy and his associates (1984a, b, 1985, 1989) based on a similar approach as Levinson (1980), resulting in a parabolic transverse shear strain distribution in thickness. This approach had been extended to investigate the numerical aspects and effects of various boundary conditions on free vibration of thick plates (Lim et al., 1998a,b) and singly and doubly curved shallow shells (Lim and Liew, 1995; Liew and Lim, 1996).

Similar to the hypothesis of Kirchhoff-Love plate theory, the Reissner-Mindlin first-order and LevinsonReddy higher-order plate theories do not consider transverse normal stress. Some authors have expressed concern over the validity of this hypothesis. Gould (1988) suggested that the concept of transverse inextensibility $\left(w=w_{o}\right)$ must be reviewed in analysis of thick plates and shells with transverse shear flexibility. He expressed that the inclusion of transverse shearing strains to extend the bounds to include somewhat thicker plates and shells is difficult to quantify since a true thick-plate or shell theory should account for transverse normal stress as well.

The transverse normal stress is considered in threedimensional elastic analysis of solids. However, such threedimensional elastic solutions are particularly scarce. Some of the investigations are concerned with rods and beams (Hutchinson, 1971; Hutchinson, 1981; Leissa and So, 1995a), parallelepiped (Fromme and Leissa, 1970; Hutchinson and Zillmer, 1983; Leissa and Zhang, 1983; Liew et al., 1995a), solid and hollow cylinders (Hutchinson, 1967; Hutchinson, 1980; Leissa and So, 1995b; Liew et al., 1995b; So and Leissa, 1997), truncated hollow cones (Leissa and So, 1995c), and open shells (Lim et al., 1998c).

To the author's knowledge, direct comparison of threedimensional elasticity solutions including and excluding transverse normal stress is only available in Hutchinson $(1979,1984)$. In these two papers, Hutchinson analyzed the 


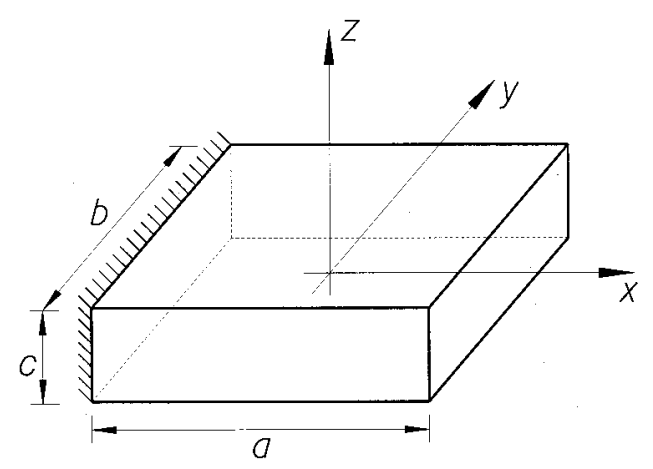

FIG. 1. Geometry of a rectangular parallelepiped.

vibration of thick, free circular plates using the Mathieu series solution and modified Pickett method to obtain exact solutions. The approximate solutions were obtained by the Mindlin thick-plate model including shear deformation and rotary inertia. These analyses concluded that, for thick, free circular plates, the approximate solution yields frequencies of sufficient accuracy for most engineering application within the range of applicability of the approximate theory. However, results are not available for thick rectangular plates. It is the key objective of this paper to address the validity of the hypothetical neglect of transverse normal stress and transverse inextensibility in the specific case of free vibration of a cantilevered rectangular parallelepiped. The assumption of transversely inextensible displacement through thickness will also be examined. This paper also intends to determine to what extent the omission of transverse normal stress in the Kirchhoff-Love thin-plate theory is applicable to cantilevered thick-plate vibrations.

\section{FORMULATION}

\section{A. Basic definition}

An isotropic parallelepiped of length $a$, width $b$, and thickness $c$ is illustrated in Fig. 1. With respect to a Cartesian coordinate system with origin located at the center of the body, a point within the body is designated by $(x, y, z)$. The parallelepiped is clamped at a surface $x=-a / 2$ with all other surfaces free.

\section{B. Three-dimensional strain and kinetic energy expressions}

For linear, elastic free vibration, the strain energy of a three-dimensional solid is

$$
\begin{aligned}
U= & \frac{1}{2} \iiint_{V}\left[(\Delta+2 G)\left(\epsilon_{x x}^{2}+\epsilon_{y y}^{2}+\epsilon_{z z}^{2}\right)+2 \Delta\left(\epsilon_{x x} \epsilon_{y y}\right.\right. \\
& \left.\left.+\epsilon_{y y} \epsilon_{z z}+\epsilon_{z z} \epsilon_{x x}\right)+G\left(\gamma_{y z}^{2}+\gamma_{z x}^{2}+\gamma_{x y}^{2}\right)\right] d x d y d z,
\end{aligned}
$$

where $V$ is the volume, $G$ is the shear modulus and

$$
\Delta=\frac{\nu E}{(1+\nu)(1-2 \nu)},
$$

$$
G=\frac{E}{2(1+\nu)},
$$

in which $E$ is the Young's modulus.

Let the displacements in the $x, y, z$ directions be $u, \mathrm{v}, w$, respectively. The normal and shear strain-displacement relations are

$$
\begin{aligned}
& \epsilon_{x x}=\frac{\partial u}{\partial x}, \\
& \epsilon_{y y}=\frac{\partial \mathrm{v}}{\partial y}, \\
& \epsilon_{z z}=\frac{\partial w}{\partial z}, \\
& \gamma_{y z}=\frac{\partial \mathrm{v}}{\partial z}+\frac{\partial w}{\partial y}, \\
& \gamma_{z x}=\frac{\partial w}{\partial x}+\frac{\partial u}{\partial z}, \\
& \gamma_{x y}=\frac{\partial u}{\partial y}+\frac{\partial \mathrm{v}}{\partial x} .
\end{aligned}
$$

The kinetic energy is

$$
T=\frac{\rho}{2} \iiint_{V}\left[\left(\frac{\partial u}{\partial t}\right)^{2}+\left(\frac{\partial \mathrm{v}}{\partial t}\right)^{2}+\left(\frac{\partial w}{\partial t}\right)^{2}\right] d V,
$$

where $\rho$ is the mass density per unit volume.

For small deformation vibration, the displacement components assume temporal simple harmonic functions in the forms of

$$
\begin{aligned}
& u(\bar{x}, \bar{y}, \bar{z}, t)=U(\bar{x}, \bar{y}, \bar{z}) \sin \omega t, \\
& \mathrm{v}(\bar{x}, \bar{y}, \bar{z}, t)=V(\bar{x}, \bar{y}, \bar{z}) \sin \omega t, \\
& w(\bar{x}, \bar{y}, \bar{z}, t)=W(\bar{x}, \bar{y}, \bar{z}) \sin \omega t,
\end{aligned}
$$

where $U, V, W$ are the displacement amplitude functions, $\omega$ is the angular frequency of vibration, and

$$
\begin{aligned}
& \bar{x}=\frac{x}{a}, \\
& \bar{y}=\frac{y}{b}, \\
& \bar{z}=\frac{z}{c},
\end{aligned}
$$

are the nondimensional coordinates.

For a nondissipative system, the total energy in a vibration cycle is conserved. The maximum strain and kinetic energy integral expressions $U_{\max }$ and $T_{\max }$ can be derived easily by substituting Eqs. (5a)-(5c) into Eqs. (1) and (4) and determining the extremum with respect to time $t$, as 


$$
\begin{aligned}
U_{\max }= & \frac{b c E}{a(1+\nu)} \iiint_{V}\left\{\frac{1-\nu}{2(1-2 \nu)}\left[\left(\frac{\partial U}{\partial \bar{x}}\right)^{2}+\left(\frac{a}{b}\right)^{2}\left(\frac{\partial V}{\partial \bar{y}}\right)^{2}+\left(\frac{a}{c}\right)^{2}\left(\frac{\partial W}{\partial \bar{z}}\right)^{2}\right]+\frac{\nu}{1-2 \nu}\left[\frac{a}{b} \frac{\partial U}{\partial \bar{x}} \frac{\partial V}{\partial \bar{y}}+\frac{a^{2}}{b c} \frac{\partial V}{\partial \bar{y}} \frac{\partial W}{\partial \bar{z}}+\frac{a}{c} \frac{\partial U}{\partial \bar{x}} \frac{\partial W}{\partial \bar{z}}\right]\right. \\
& +\frac{1}{4}\left[\left(\frac{a}{c}\right)^{2}\left(\frac{\partial V}{\partial \bar{z}}\right)^{2}+\frac{2 a^{2}}{b c} \frac{\partial V}{\partial \bar{z}} \frac{\partial W}{\partial \bar{y}}+\left(\frac{a}{b}\right)^{2}\left(\frac{\partial W}{\partial \bar{y}}\right)^{2}+\left(\frac{a}{c}\right)^{2}\left(\frac{\partial U}{\partial \bar{z}}\right)^{2}+\frac{2 a}{c} \frac{\partial U}{\partial \bar{z}} \frac{\partial W}{\partial \bar{x}}+\left(\frac{\partial W}{\partial \bar{x}}\right)^{2}+\left(\frac{a}{b}\right)^{2}\left(\frac{\partial U}{\partial \bar{y}}\right)^{2}\right. \\
& \left.\left.+\frac{2 a}{b} \frac{\partial U}{\partial \bar{y}} \frac{\partial V}{\partial \bar{x}}+\left(\frac{\partial V}{\partial \bar{x}}\right)^{2}\right]\right\} d \bar{x} d \bar{y} d \bar{z}
\end{aligned}
$$

and

$$
T_{\max }=\frac{\rho \omega^{2} a b c}{2} \iiint_{V}\left(U^{2}+V^{2}+W^{2}\right) d \bar{x} d \bar{y} d \bar{z} .
$$

\section{Three-dimensional energy functional and eigenvalue equation}

The displacement amplitude functions for a vibrating parallelepiped can be expressed by a set of three-dimensional (3D) $p$-Ritz functions. These functions are the products of 2D $p$-Ritz functions $\phi_{u}(\bar{x}, \bar{y}), \phi_{\mathrm{v}}(\bar{x}, \bar{y}), \phi_{w}(\bar{x}, \bar{y})$ for the midsurface deformation, and 1D $p$-Ritz functions $\psi_{u}(\bar{z})$, $\psi_{\mathrm{v}}(\bar{z}), \psi_{w}(\bar{z})$ for the thickness deformation. The displacement amplitude functions are

$$
\begin{aligned}
& U(\bar{x}, \bar{y}, \bar{z})=\sum_{i=1}^{m} \sum_{j=1}^{n} C_{u}^{i j} \phi_{u}^{i}(\bar{x}, \bar{y}) \psi_{u}^{j}(\bar{z}), \\
& V(\bar{x}, \bar{y}, \bar{z})=\sum_{i=1}^{m} \sum_{j=1}^{n} C_{\mathrm{v}}^{i j} \phi_{\mathrm{v}}^{i}(\bar{x}, \bar{y}) \psi_{\mathrm{v}}^{j}(\bar{z}), \\
& W(\bar{x}, \bar{y}, \bar{z})=\sum_{i=1}^{m} \sum_{j=1}^{n} C_{w}^{i j} \phi_{w}^{i}(\bar{x}, \bar{y}) \psi_{w}^{j}(\bar{z}),
\end{aligned}
$$

in which $C_{u}^{i j}, C_{\mathrm{v}}^{i j}$, and $C_{w}^{i j}$ are the unknown coefficients.

An energy functional is defined as the difference of the maximum strain and kinetic energy components

$$
\Pi=U_{\max }-T_{\max } .
$$

Numerical frequency solutions can be obtained by minimizing this energy functional with respect to the unknown coefficients in accordance with the Ritz procedure

$$
\frac{\partial \Pi}{\partial C_{\alpha}^{i j}}=0 ; \quad \alpha=u, \quad \mathrm{v}, \quad \text { and } w,
$$

which leads to the governing eigenvalue equation

$$
\left(K-\lambda^{2} M\right)\{C\}=\{0\},
$$

where

$$
\lambda=\omega a \sqrt{\frac{\rho(1+\nu)}{E}}
$$

is the dimensionless frequency parameter. The derivatives of $U_{\max }$ and $T_{\max }$ with respect to the unknown coefficients are presented in Appendix A.

The stiffness and mass matrices are

$$
\begin{aligned}
& K=\left[\begin{array}{lll}
\boldsymbol{k}_{u u} & \boldsymbol{k}_{u \mathrm{v}} & \boldsymbol{k}_{u w} \\
& \boldsymbol{k}_{\mathrm{vv}} & \boldsymbol{k}_{\mathrm{v} w} \\
\mathrm{sym} & & \boldsymbol{k}_{w w}
\end{array}\right], \\
& M=\left[\begin{array}{lll}
\boldsymbol{m}_{u u} & {[0]} & {[0]} \\
& \boldsymbol{m}_{\mathrm{vv}} & {[0]} \\
\operatorname{sym} & & \boldsymbol{m}_{w w}
\end{array}\right],
\end{aligned}
$$

and the vector of unknown coefficients is

$$
C=\left\{\begin{array}{l}
\left\{\boldsymbol{C}_{u}\right\} \\
\left\{\boldsymbol{C}_{\mathrm{v}}\right\} \\
\left\{\boldsymbol{C}_{w}\right\}
\end{array}\right\} .
$$

The elements in the stiffness submatrix are

$$
\begin{aligned}
k_{u u}^{i k j l}= & \frac{1-\nu}{1-2 \nu} I_{\phi_{u u}^{i k}}^{1010} J_{\psi_{u u}^{j l}}^{00}+\frac{1}{2}\left[\left(\frac{a}{c}\right)^{2} I_{\phi_{u u}^{i k}}^{0000} J_{\psi_{u u}^{1 j}}^{11}\right. \\
& \left.+\left(\frac{a}{b}\right)^{2} I_{\phi_{u u}^{i k}}^{0101} J_{\psi_{u u}^{j l}}^{00}\right], \\
k_{u \mathrm{~V}}^{i k j l}= & \frac{\nu}{1-2 \nu} \frac{a}{b} I_{\phi_{u \mathrm{v}}^{i k}}^{1001} J_{\psi_{u \mathrm{v}}^{j l}}^{00}+\frac{a}{2 b} I_{\phi_{u \mathrm{~V}}^{01 k}}^{010} J_{\psi_{u \mathrm{~V}}^{j l}}^{00},
\end{aligned}
$$

$$
k_{u w}^{i k j l}=\frac{\nu}{1-2 \nu} \frac{a}{c} I_{\phi_{u w}^{i k}}^{1000} J_{\psi_{u w}^{j l}}^{01}+\frac{a}{2 c} I_{\phi_{u w}^{i k}}^{0010} J_{\psi_{u w}^{j l}}^{10},
$$

$$
\begin{aligned}
k_{\mathrm{vv}}^{i k j l}= & \frac{1-\nu}{1-2 \nu}\left(\frac{a}{b}\right)^{2} I_{\phi_{\mathrm{vv}}^{i k}}^{0101} J_{\psi_{\mathrm{vv}}^{j l}}^{00}+\frac{1}{2}\left[\left(\frac{a}{c}\right)^{2} I_{\phi_{\mathrm{vv}}^{i k}}^{0000} J_{\psi_{\mathrm{vv}}^{j l}}^{11}\right. \\
& \left.+I_{\phi_{\mathrm{vv}}^{i k}}^{1010} J_{\psi_{\mathrm{vv}}^{j l}}^{00}\right]
\end{aligned}
$$

$$
k_{\mathrm{v} w}^{i k j l}=\frac{\nu}{1-2 \nu} \frac{a^{2}}{b c} I_{\phi_{\mathrm{v} w}^{i k}}^{0100} J_{\psi_{\mathrm{v} w}^{j l}}^{01}+\frac{a^{2}}{2 b c} I_{\phi_{\mathrm{v} w}^{i k}}^{0001} J_{\psi_{\mathrm{v} w}^{j l}}^{10},
$$

$$
\begin{aligned}
k_{w w}^{i k j l}= & \frac{1-\nu}{1-2 \nu}\left(\frac{a}{c}\right)^{2} I_{\phi_{w w}^{i k}}^{0000} J_{\psi_{w w}^{j l}}^{11}+\frac{1}{2}\left[\left(\frac{a}{b}\right)^{2} I_{\phi_{w w}^{i k}}^{0101} J_{\psi_{w w}^{j l}}^{00}\right. \\
& \left.+I_{\phi_{w w}^{i k}}^{1010} J_{\psi_{w w}^{j l}}^{00}\right],
\end{aligned}
$$

and the elements in the mass submatrix are

$$
\begin{aligned}
& m_{u u}^{i k j l}=I_{\phi_{u u}^{i k}}^{0000} J_{\psi_{u u}^{j l}}^{00}, \\
& m_{\mathrm{vv}}^{i k j l}=I_{\phi_{\mathrm{vv}}^{i k}}^{0000} J_{\psi_{\mathrm{vv}}}^{00},
\end{aligned}
$$




$$
m_{w w}^{i k j l}=I_{\phi_{w w}^{i k}}^{0000} J_{w w}^{00}
$$

in which

$$
\begin{aligned}
I_{\phi_{\alpha \beta}^{i k}}^{a b c d} & =\iint_{\bar{A}} \frac{\partial^{a+b} \phi_{\alpha}^{i}(\bar{x}, \bar{y})}{\partial \bar{x}^{a} \partial \bar{y}^{b}} \frac{\partial^{c+d} \phi_{\beta}^{k}(\bar{x}, \bar{y})}{\partial \bar{x}^{c} \partial \bar{y}^{d}} d \bar{x} d \bar{y}, \\
J_{\psi_{\alpha \beta}^{j l}}^{e f} & =\int_{\bar{c}} \frac{\partial^{e} \psi_{\alpha}^{j}(\bar{z})}{\partial \bar{z}^{e}} \frac{\partial^{f} \psi_{\beta}^{l}(\bar{z})}{\partial \bar{z}^{f}} d \bar{z},
\end{aligned}
$$

where $\alpha, \beta=u, \mathrm{v}, w ; i, k=1,2, \ldots, m$, and $m$ is the total number of terms employed in the two-dimensional $p$-Ritz shape functions for planes parallel to the $x y$-midsurface; $j, l=1,2, \ldots, n$, and $n$ is the total number of terms employed in the one-dimensional $p$-Ritz shape functions in the thickness $z$-direction. The normalized midsurface area is denoted as $\bar{A}$ and the normalized thickness is $\bar{c}$.

\section{Boundary conditions and $p$-Ritz admissible functions}

In the Ritz method, we ensure the satisfaction of geometric boundary conditions such as displacements and rotations at the boundary surfaces. Although satisfaction of natural boundary conditions such as shear forces and moments is not required at the outset, accurate computation shows that stresses at the free boundaries approach zero if accurate frequencies (for vibration) or buckling loads (for buckling) are obtained. For a parallelepiped, no geometric boundary condition is required for a free boundary surface. For a clamped boundary surface at $\bar{x}=-0.5$, the geometric boundary conditions are

$\left.U(\bar{x}, \bar{y}, \bar{z})\right|_{\bar{x}=-0.5}=\left.V(\bar{x}, \bar{y}, \bar{z})\right|_{\bar{x}=-0.5}=\left.W(\bar{x}, \bar{y}, \bar{z})\right|_{\bar{x}=-0.5}=0$.

The displacement components denoted by $U(\bar{x}, \bar{y}, \bar{z})$, $V(\bar{x}, \bar{y}, \bar{z})$, and $W(\bar{x}, \bar{y}, \bar{z})$ are truncated finite series expressed in Eqs. (5a)-(5c). The two-dimensional deformation admissible functions $\phi_{u}(\bar{x}, \bar{y}), \phi_{\mathrm{v}}(\bar{x}, \bar{y})$, and $\phi_{w}(\bar{x}, \bar{y})$ are geometrically compliant polynomial functions derived such that the geometric boundary conditions (20) are satisfied at the outset (Lim et al., 1998a). They are composed of the product of a series of simple two-dimensional polynomials $F_{\phi}(\bar{x}, \bar{y})$ and boundary-compliant basic functions $\phi_{u}^{b}(\bar{x}, \bar{y}), \phi_{\mathrm{v}}^{b}(\bar{x}, \bar{y})$, and $\phi_{w}^{b}(\bar{x}, \bar{y})$. The basic functions are geometric expressions of the parallelepiped boundaries raised to an appropriate basic power in accordance with various boundary constraints (Lim et al., 1998a). For a cantilevered parallelepiped clamped at $\bar{x}=-0.5$, the two-dimensional deformation admissible functions are

$$
\begin{aligned}
& \phi_{u}(\bar{x}, \bar{y})=(\bar{x}+0.5) F_{\phi}(\bar{x}, \bar{y}), \\
& \phi_{\mathrm{v}}(\bar{x}, \bar{y})=(\bar{x}+0.5) F_{\phi}(\bar{x}, \bar{y}), \\
& \phi_{w}(\bar{x}, \bar{y})=(\bar{x}+0.5) F_{\phi}(\bar{x}, \bar{y}),
\end{aligned}
$$

where

$$
F_{\phi}(\bar{x}, \bar{y})=\sum_{q=0}^{p_{x y}} \sum_{i=0}^{q} \bar{x}^{q-i} \bar{y}^{i},
$$

in which $p_{x y}$ is the highest degree of the two-dimensional polynomial in the admissible functions and $m=\left(p_{x y}+1\right)$ $\times\left(p_{x y}+2\right) / 2$ is the number of the $2 \mathrm{D}$ terms.

Similarly, the one-dimensional thickness admissible functions $\psi_{u}(\bar{z}), \psi_{\mathrm{v}}(\bar{z})$, and $\psi_{w}(\bar{z})$ are the products of sets of one-dimensional polynomial functions $F_{\psi}(\bar{z})$ and appropriate basic functions $\psi_{u}^{b}(\bar{z}), \psi_{\mathrm{v}}^{b}(\bar{z})$, and $\psi_{w}^{b}(\bar{z})$. For a cantilevered parallelepiped clamped at $\bar{x}=-0.5$, the onedimensional thickness deformation admissible functions are

$$
\begin{aligned}
& \psi_{u}(\bar{z})=F_{\psi}(\bar{z}), \\
& \psi_{\mathrm{v}}(\bar{z})=F_{\psi}(\bar{z}), \\
& \psi_{w}(\bar{z})=F_{\psi}(\bar{z}),
\end{aligned}
$$

where

$$
F_{\psi}(\bar{z})=\sum_{i=0}^{p_{z}} \bar{z}^{i},
$$

in which $p_{z}$ is the highest degree of the one-dimensional polynomial in the admissible functions and $n=p_{z}+1$ indicates the number of $1 \mathrm{D}$ terms.

\section{RESULTS AND DISCUSSION}

\section{A. Mode classification and convergence of eigenvalues}

To improve computational efficiency without sacrificing numerical accuracy, classification of vibration modes is possible by grouping terms with odd and even powers of $\bar{x}, \bar{y}$ and $\bar{z}$ in $F_{\phi}(\bar{x}, \bar{y})$, and $F_{\psi}(\bar{z})$ in Eqs. (22) and (24), respectively. This tremendously reduces the number of terms in each series and thus the determinant size of the eigenvalue equation is considerably smaller. Huge computational effort can be saved without affecting the numerical accuracy because the odd and even terms of $\bar{x}, \bar{y}$, and $\bar{z}$ only contribute to specific modes and they are trivial in other modes (Lim et al., 1998a).

Classification of vibration modes depends on symmetry of geometry and boundary conditions. Eight mode classes are possible for a parallelepiped with perfect symmetry in geometry and boundary conditions. For a cantilevered parallelepiped, mode classification can be referred to the $x y$ - and $x z$-planes (see Fig. 1) perpendicular to the clamped surface at $\bar{x}=-0.5$. Four mode classes exist as the symmetricsymmetric (SS), symmetric-antisymmetric (SA), antisymmetric-symmetric (AS), and antisymmetricantisymmetric (AA) modes.

Convergence of eigenvalues presented in Table I for a cantilevered cube is investigated with respect to increases in $p_{z}$ and $p_{x y}$ for the $1 \mathrm{D}$ and $2 \mathrm{D}$ polynomial functions, respectively, in accordance with various symmetry classes. The polynomial degrees are increased from 7 to 10 for $p_{x y}$ and from 7 to 9 for $p_{z}$. As observed in Table I, downward convergence is obvious. It is a unique numerical feature of the 
TABLE I. Convergence of $\lambda=\omega a \sqrt{\rho / E}$ for a cantilevered cube with $\nu$ $=0.3$.

\begin{tabular}{|c|c|c|c|c|c|}
\hline \multirow{2}{*}{$\begin{array}{c}\text { Symmetry } \\
\text { Class }\end{array}$} & \multirow[b]{2}{*}{$p_{x y} \times p_{z}$} & \multicolumn{4}{|c|}{ Mode number } \\
\hline & & M-1 & M-2 & M-3 & M-4 \\
\hline \multirow[t]{6}{*}{ SS } & $7 \times 7$ & 1.5977 & 2.5805 & 2.9126 & 3.0508 \\
\hline & $8 \times 7$ & 1.5972 & 2.5805 & 2.9123 & 3.0508 \\
\hline & $9 \times 7$ & 1.5969 & 2.5804 & 2.9120 & 3.0507 \\
\hline & $10 \times 7$ & 1.5967 & 2.5803 & 2.9119 & 3.0507 \\
\hline & $10 \times 8$ & 1.5965 & 2.5803 & 2.9118 & 3.0507 \\
\hline & $10 \times 9$ & 1.5965 & 2.5803 & 2.9118 & 3.0507 \\
\hline \multirow[t]{6}{*}{ SA } & $7 \times 7$ & 0.66851 & 1.7671 & 2.7523 & 3.0578 \\
\hline & $8 \times 7$ & 0.66834 & 1.7670 & 2.7523 & 3.0575 \\
\hline & $9 \times 7$ & 0.66824 & 1.7669 & 2.7522 & 3.0573 \\
\hline & $10 \times 7$ & 0.66816 & 1.7669 & 2.7522 & 3.0573 \\
\hline & $10 \times 8$ & 0.66796 & 1.7668 & 2.7521 & 3.0567 \\
\hline & $10 \times 9$ & 0.66796 & 1.7668 & 2.7521 & 3.0567 \\
\hline \multirow[t]{6}{*}{ AS } & $7 \times 7$ & 0.67102 & 1.7696 & 2.7529 & 3.0670 \\
\hline & $8 \times 7$ & 0.66993 & 1.7679 & 2.7525 & 3.0629 \\
\hline & $9 \times 7$ & 0.66927 & 1.7675 & 2.7523 & 3.0597 \\
\hline & $10 \times 7$ & 0.66885 & 1.7672 & 2.7523 & 3.0589 \\
\hline & $10 \times 8$ & 0.66885 & 1.7672 & 2.7523 & 3.0589 \\
\hline & $10 \times 9$ & 0.66881 & 1.7672 & 2.7522 & 3.0589 \\
\hline \multirow[t]{6}{*}{$\mathrm{AA}$} & $7 \times 7$ & 0.90909 & 2.1790 & 2.6909 & 2.7474 \\
\hline & $8 \times 7$ & 0.90853 & 2.1788 & 2.6879 & 2.7467 \\
\hline & $9 \times 7$ & 0.90832 & 2.1786 & 2.6870 & 2.7465 \\
\hline & $10 \times 7$ & 0.90821 & 2.1786 & 2.6865 & 2.7464 \\
\hline & $10 \times 8$ & 0.90821 & 2.1786 & 2.6865 & 2.7464 \\
\hline & $10 \times 9$ & 0.90815 & 2.1786 & 2.6863 & 2.7463 \\
\hline
\end{tabular}

Ritz procedure which overestimates vibration frequency and buckling load and underestimates bending deflection. Convergence of eigenvalues can be ensured by including an adequate number of terms in the admissible shape functions. As seen in Table I, the eigenvalues are converged to at least three significant figures and in most cases more than three significant figures.

\section{B. Comparison of solutions}

As described in the Introduction, the Kirchhoff-Love thin-plate theory and most of the existing thick-plate models neglect transverse normal stress $\sigma_{z z}$. Although the omission of $\sigma_{z z}$ for thin-plate theory has been examined and verified in many publications (Leissa, 1969), it is rather hypothetical in Reissner-Mindlin and Levinson-Reddy thick-plate models and has been questioned by a number of researchers such as Gould (1988). These thick-plate models further assume constant transverse normal displacement $(w)$ through the thickness, or transverse inextensibility. It is easily verified that a trivial $\sigma_{z z}$ implies

$$
\epsilon_{z z}=-\frac{\nu}{1-\nu}\left(\epsilon_{x x}+\epsilon_{y y}\right) \text {, }
$$

in accordance with the generalized Hooke's law. Therefore, to neglect $\sigma_{z z}$ and at the same time keep $w$ constant through the thickness (transverse inextensibility) is an inconsistent thick-plate model. Although some early higher-order thickplate models (Whitney and Sun, 1973, 1974; Iyengar et al., 1974 and Lo et al., 1977a,b) had suggested nonlinear (quadratic and cubic) expressions for $w$, these models had not gained much popularity over the Reissner-Mindlin and Levinson-Reddy thick-plate models. It is, therefore, the pri- mary objective of this paper to address the importance of $\sigma_{z z}$ and the consistency of a thick-plate model with nonlinear $w$.

To simulate a thick-plate model neglecting $\sigma_{z z}$, Eq. (25) is substituted into Eq. (1) to derive the strain energy as

$$
\begin{aligned}
U= & \frac{1}{2} \iiint_{V}\left[\frac{E}{1-\nu^{2}}\left(\epsilon_{x x}^{2}+\epsilon_{y y}^{2}\right)+\frac{2 \nu E}{1-\nu^{2}} \epsilon_{x x} \epsilon_{y y}\right. \\
& \left.+G\left(\gamma_{y z}^{2}+\gamma_{z x}^{2}+\gamma_{x y}^{2}\right)\right] d x d y d z,
\end{aligned}
$$

while the kinetic energy expression (4) is still valid. The maximum strain energy is presented in Appendix B. The formulation from Eqs. (5a)-(16) is repeated to obtain a corresponding eigenvalue equation. The stiffness matrix element expressions for a parallelepiped neglecting $\sigma_{z z}$ are presented in Appendix C.

For the purpose of comparison and to simulate constant $w$ through thickness in Reissner-Mindlin and LevinsonReddy thick-plate models, $\psi_{w}(\bar{z})=F_{\psi}(\bar{z})=1$ is set in Eq. (23c) for solutions with $\sigma_{z z} \approx 0$ such that the transverse normal displacement

$$
W(\bar{x}, \bar{y}, \bar{z})=W(\bar{x}, \bar{y})=\sum_{i=1}^{m} C_{w}^{i 1} \phi_{w}^{i}(\bar{x}, \bar{y})
$$

in Eq. (9c) is independent of $\bar{z}$.

A comparison of free vibration frequency solutions with the results of Leissa and Zhang (1983) (denoted by A) is presented in Table II for parallelepipeds with various aspect ratios. The vibration frequencies are classified into four symmetry classes with respect to $x y$ - and $x z$-planes. Solutions (denoted by $\mathrm{B}$ ) by solving the full three-dimensional energy functional using the Ritz energy approach as governed by Eq. (12) are presented. In addition, solutions (denoted by C) neglecting $\sigma_{z z}$ and assuming constant $w$ through thickness with reference to Eqs. (26) and (27) are also included. Overall, excellent agreement of solutions between $\mathrm{A}$ and $\mathrm{B}$ is observed, while the agreement with respect to $\mathrm{C}$ is generally satisfactory. The excellent agreement of A and B solutions is expected, as Leissa and Zhang (1983) also solved the full three-dimensional energy functional. The most obvious discrepancy between solutions of A and C or B and C happens in the SS modes for a cantilevered parallelepiped with $a / b$ $=0.5$ and $b / c=1$. One of the reasons the largest discrepancy is observed in this case is because this parallelepiped has a thickness twice the length $(c / a=2)$ and the effect of $\sigma_{z z}$ is expected to be more obvious as the thickness increases. The other parallelepiped configurations have $c / a$ either 0.5 or 1 . We will see the effects of $\sigma_{z z}$ more closely in the next section.

\section{Effects of transverse normal stress $\sigma_{z z}$}

A set of first-known solutions for examining the effects of $\sigma_{z z}$ for various parallelepiped configurations is presented. Figures $2-5$ present the nondimensional vibration frequency parameters $\lambda$ with varying thickness ratio $c / b$ in four distinct mode classes for a cantilevered rectangular parallelepiped with aspect ratio $a / b=0.5$. 
TABLE II. Comparison of $\lambda=\omega a \sqrt{\rho / E}$ for cantilevered rectangular parallelepiped ( $\nu=0.3)$ with $x y$ - and $x z$-symmetric planes.

\begin{tabular}{|c|c|c|c|c|c|c|c|c|c|c|c|c|c|c|}
\hline \multicolumn{2}{|c|}{ Aspect ratios } & \multirow[b]{2}{*}{ Sources } & \multicolumn{12}{|c|}{ Mode frequencies } \\
\hline$a / b$ & $b / c$ & & SS-1 & SS-2 & SS-3 & SA-1 & SA-2 & SA-3 & AS-1 & AS-2 & AS-3 & AA-1 & AA-2 & AA-3 \\
\hline \multirow[t]{3}{*}{1} & 1 & A & 1.6000 & 2.5812 & 2.9154 & 0.67087 & 1.7695 & 2.7562 & 0.67087 & 1.7695 & 2.7562 & 0.90930 & 2.1801 & 2.7011 \\
\hline & & B & 1.5965 & 2.5803 & 2.9118 & 0.66796 & 1.7668 & 2.7521 & 0.66881 & 1.7672 & 2.7522 & 0.90815 & 2.1786 & 2.6863 \\
\hline & & $\mathrm{C}$ & 1.5800 & 2.8160 & 3.2228 & 0.66226 & 1.7595 & 2.7518 & 0.65893 & 1.7726 & 3.0386 & 0.90942 & 2.1982 & 2.7132 \\
\hline \multirow[t]{3}{*}{2} & 1 & A & 1.5938 & 4.5811 & 5.0646 & 0.44371 & 1.6711 & 3.7237 & 0.44371 & 1.6711 & 3.7237 & 0.90402 & 2.7191 & 4.1787 \\
\hline & & B & 1.5888 & 4.5496 & 5.0520 & 0.44021 & 1.6612 & 3.4626 & 0.44072 & 1.6629 & 3.4654 & 0.90208 & 2.7012 & 4.1711 \\
\hline & & $\mathrm{C}$ & 1.5778 & 4.5898 & 5.2746 & 0.43672 & 1.6516 & 3.4475 & 0.43504 & 1.6592 & 3.4757 & 0.90254 & 2.7082 & 4.1953 \\
\hline \multirow[t]{3}{*}{1} & 2 & A & 1.5962 & 2.7974 & 3.1994 & 0.44733 & 1.6642 & 2.2777 & 0.66744 & 1.7744 & 3.0680 & 0.78831 & 2.2196 & 3.4387 \\
\hline & & B & 1.5920 & 2.7959 & 3.1946 & 0.44413 & 1.6551 & 2.2733 & 0.66496 & 1.7721 & 3.0436 & 0.78586 & 2.2094 & 3.4007 \\
\hline & & $\mathrm{C}$ & 1.5800 & 2.8160 & 3.2228 & 0.44030 & 1.6455 & 2.2634 & 0.65893 & 1.7726 & 3.0386 & 0.78453 & 2.2063 & 3.3960 \\
\hline \multirow[t]{3}{*}{0.5} & 1 & A & 1.4670 & 1.5623 & 1.7967 & 0.83004 & 1.5317 & 1.7647 & 0.83004 & 1.5317 & 1.7647 & 0.91636 & 1.3550 & 1.9061 \\
\hline & & B & 1.4679 & 1.5588 & 1.8423 & 0.82732 & 1.5293 & 1.7628 & 0.82831 & 1.5289 & 1.7629 & 0.91485 & 1.3530 & 1.9052 \\
\hline & & $\mathrm{C}$ & 1.5281 & 1.6889 & 2.0438 & 0.82618 & 1.5281 & 1.7523 & 0.81863 & 1.6839 & 2.3306 & 0.91870 & 1.3646 & 1.8903 \\
\hline \multirow[t]{3}{*}{0.5} & 2 & A & 1.5325 & 1.6835 & 2.0337 & 0.67484 & 1.3538 & 1.8070 & 0.82712 & 1.6911 & 2.3128 & 0.82131 & 1.7307 & 2.1420 \\
\hline & & B & 1.5300 & 1.6812 & 2.0282 & 0.67200 & 1.3507 & 1.8037 & 0.82531 & 1.6892 & 2.3020 & 0.81937 & 1.7284 & 2.1363 \\
\hline & & $\mathrm{C}$ & 1.5208 & 1.6889 & 2.0438 & 0.66572 & 1.3496 & 1.7933 & 0.81863 & 1.6839 & 2.3306 & 0.81829 & 1.7263 & 2.1193 \\
\hline
\end{tabular}

${ }^{\mathrm{a}}$ Leissa and Zhang (1983).

${ }^{\mathrm{b}}$ Present (including $\sigma_{z z}$ ).

${ }^{\mathrm{c}}$ Present (excluding $\sigma_{z z}$ and assuming constant $w$ through thickness).

Frequency solutions for the first four SS modes with $c / b$ ranging from 0.2 to 2.0 are presented in Fig. 2. As expected, excellent agreement for solutions including and excluding $\sigma_{z z}$ (constant $w$ through thickness) is achieved for small thickness ratio. The discrepancy of solutions becomes more apparent as $c / b$ increases. The onsets of distinct discrepancy between the solutions are associated with smaller $c / b$ for higher vibration modes. For instance, distinct discrepancy of SS-1 solutions can be observed for $c / b>0.9$, while for SS-4 the onset reduces to $c / b \approx 0.4$. The physical implication is as follows. It is widely known that higher vibration modes pos-

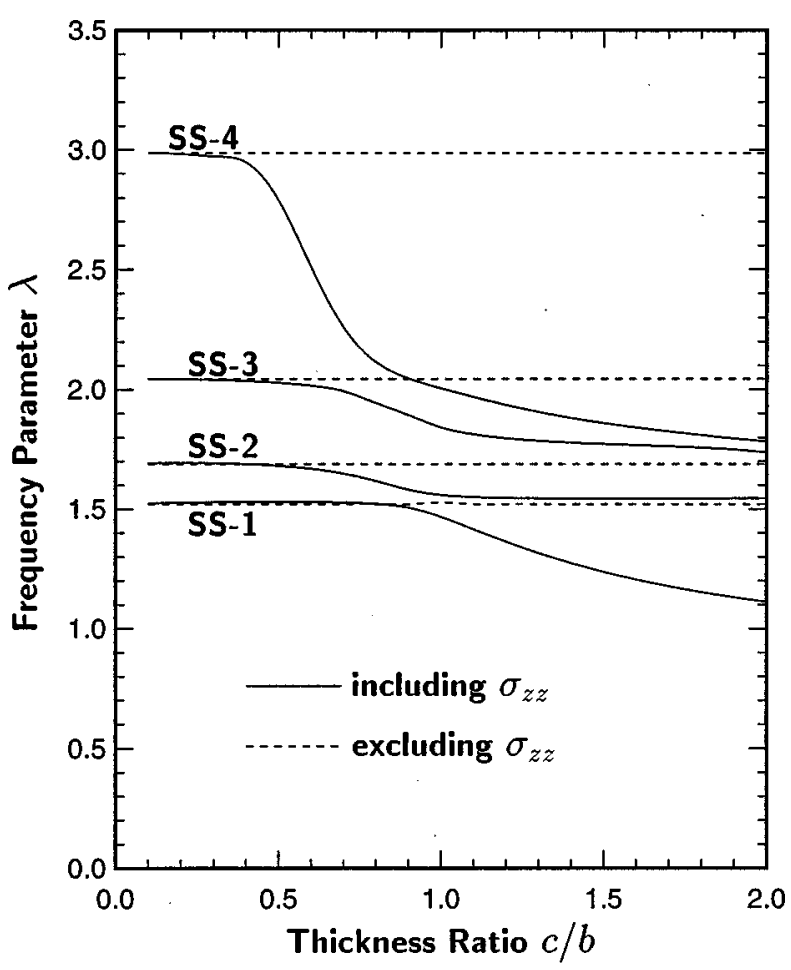

FIG. 2. Effect of thickness ratio on the SS frequencies for a thick cantilevered rectangular parallelepiped with $\nu=0.3$ and $a / b=0.5$. sess more nodal lines (Lim et al., 1998b) for plates; or more nodal surfaces for parallelepipeds. A nodal line (surface) is a line (surface) with zero vibration amplitude. We may treat a nodal line (surface) as a boundary line (surface) with certain constraints which should not be seen as a simply supported or clamped boundary constraints. As a result, the effective region is smaller for higher vibration modes with more nodal lines (surfaces) or, equivalently, the effective thickness ratio becomes larger than the overall thickness ratio $c / b$. Consequently, the onset of distinct discrepancy between solutions

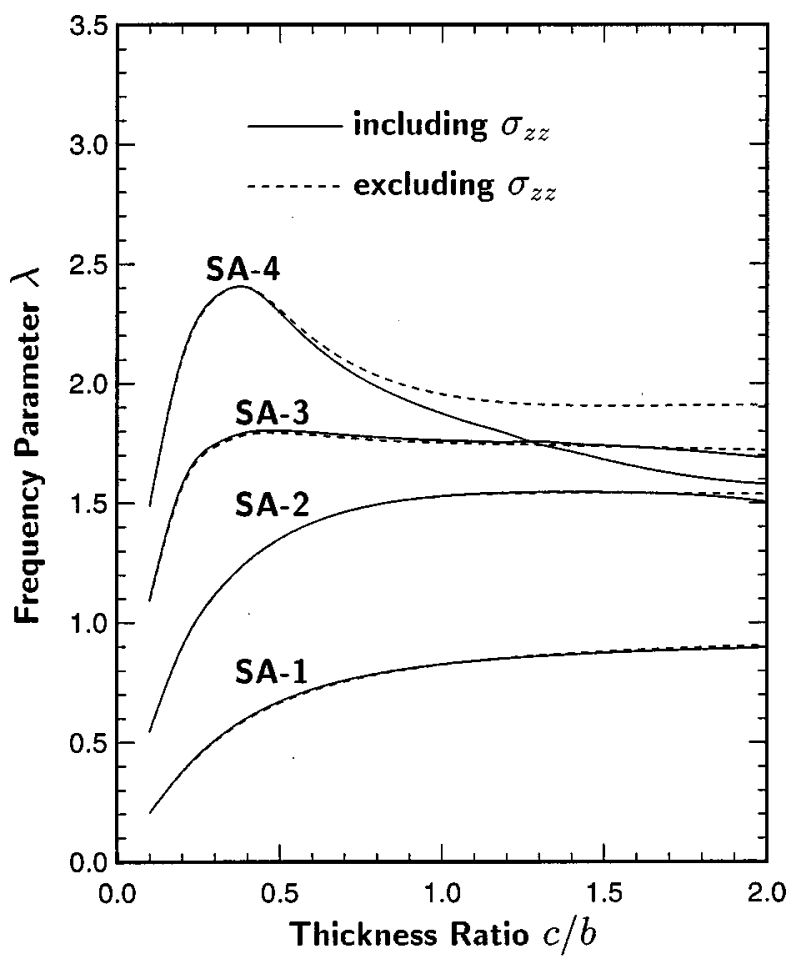

FIG. 3. Effect of thickness ratio on the SA frequencies for a thick cantilevered rectangular parallelepiped with $\nu=0.3$ and $a / b=0.5$. 


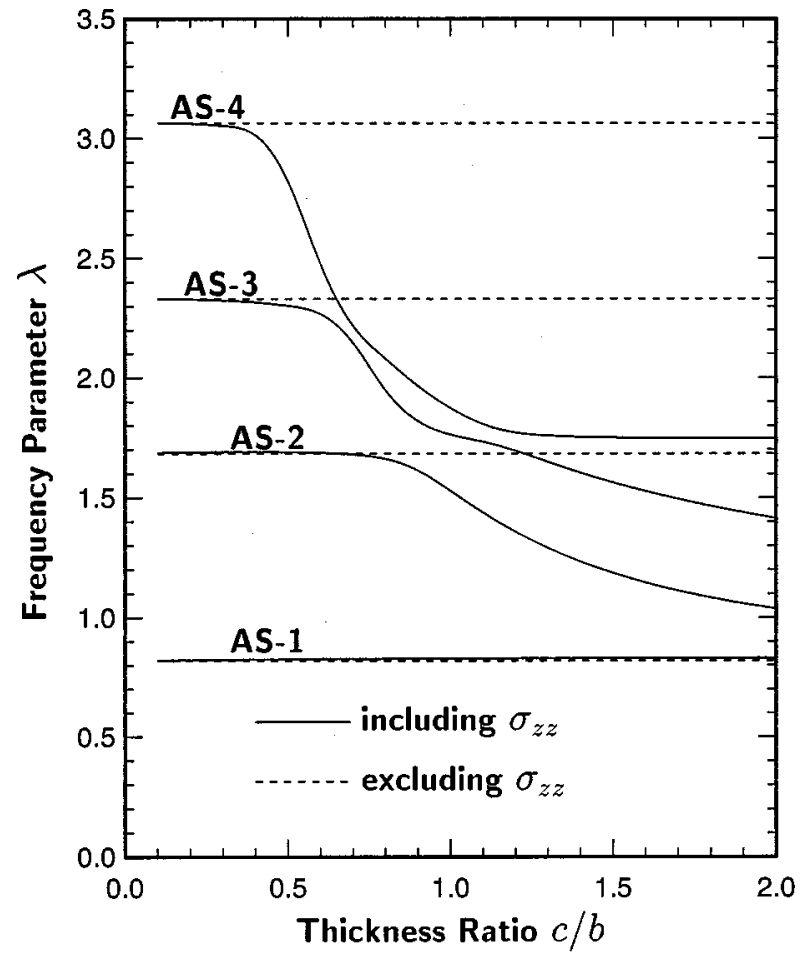

FIG. 4. Effect of thickness ratio on the AS frequencies for a thick cantilevered rectangular parallelepiped with $\nu=0.3$ and $a / b=0.5$.

with respect to $c / b$ becomes smaller for higher vibration modes. The effects of $\sigma_{z z}$ on other classes of vibration modes are illustrated in Figs. 3-5.

It is also noticed that solutions considering $\sigma_{z z}$ are always lower when the effect of $\sigma_{z z}$ becomes significant for large $c / b$. Although $\epsilon_{z z} \neq 0$, as determined by Eq. (25), even if $\sigma_{z z}$ is neglected, the consideration of $\sigma_{z z}$ allows transverse extensibility not governed by Eq. (25), thus providing a further degree of flexibility in the transverse normal direction. Vibration frequency is smaller if $\sigma_{z z}$ is considered because flexibility reduces the structural stiffness for the parallelepiped.

In Figs. 2-5, more apparent discrepancy of solutions for the SS modes compared to the other modes is observed. This is true only for a cantilevered parallelepiped and it is inconclusive for other parallelepiped configurations as the significance and effects of $\sigma_{z z}$ depend not only on geometry but also boundary conditions. However, it is reasonable to conclude that the effect of $\sigma_{z z}$ for $c / b<0.5$ is rather insignificant for some lower modes in the aspect of free vibration of thick plates and parallelepipeds. Such conclusion was also reached by Hutchinson $(1979,1984)$ in analyzing vibrations of thick, free circular plates using exact and approximate methods. These analyses concluded that, for thick, free circular plates, the approximate solution yields frequencies of sufficient accuracy for most engineering application within the range of applicability of the approximate theory. Based on the conclusions, accurate solutions employing the Levinson-Reddy higher-order plate theory have been reported (Liew and Lim, 1996; Lim and Liew, 1995, 1996; Lim et al., 1998a,b).

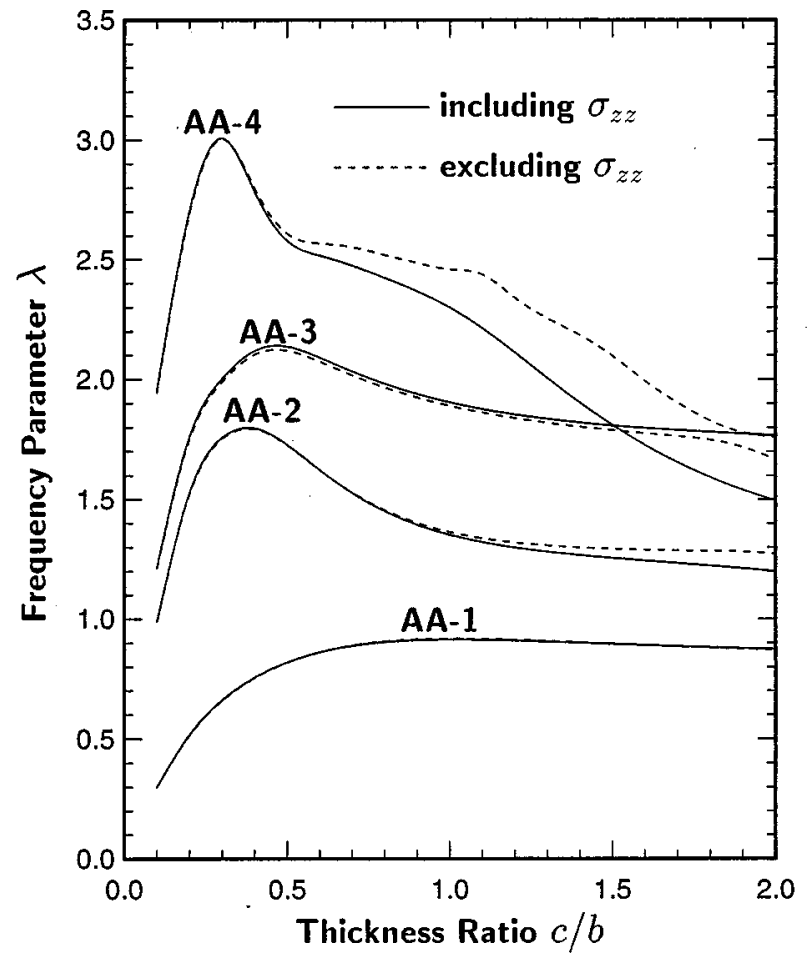

FIG. 5. Effect of thickness ratio on the AA frequencies for a thick cantilevered rectangular parallelepiped with $\nu=0.3$ and $a / b=0.5$.

\section{CONCLUSIONS}

New solutions and benchmarks for examining the effects of transverse normal stress $\sigma_{z z}$ in the context of vibration of a cantilevered parallelepiped are presented and analyzed. The hypothetical assumption of neglecting $\sigma_{z z}$ while maintaining constant transverse normal displacement $w$ through thickness in existing thick-plate models is investigated by solving the full three-dimensional energy functional. The extent to which the assumption is applicable is verified.

When the effect of transverse normal stress becomes significant for large thickness ratio, vibration frequency is always lower when transverse normal stress is considered because transverse extensibility provides a further degree of flexibility in the transverse normal direction and thus reduces the stiffness of a parallelepiped. For higher vibration modes, the onset of significant contribution of transverse normal stress is associated with a lower thickness ratio.

\section{ACKNOWLEDGMENT}

A research fellowship for the author from The University of Hong Kong is gratefully acknowledged.

\section{APPENDIX A}

The derivatives of strain and kinetic energy integrals with respect to the unknown coefficients for Eq. (11) are as follows: 


$$
\begin{aligned}
\frac{\partial U_{\max }}{\partial C_{u}^{i j}}= & \frac{b c E}{a(1+\nu)} \sum_{k=1}^{m} \sum_{l=1}^{n}\left\{\frac{1-\nu}{1-2 \nu} C_{u}^{k l} I_{\phi_{u u}^{i k}}^{1010} J_{\psi_{u u}^{0 j}}^{00}\right. \\
& +\frac{\nu}{1-2 \nu}\left[\frac{a}{b} C_{\mathrm{v}}^{k l} I_{\phi_{u \mathrm{v}}^{i k}}^{1001} J_{\psi_{u \mathrm{v}}^{j l}}^{00}+\frac{a}{c} C_{w}^{k l} I_{\phi_{u w}^{i k}}^{1000} J_{\psi_{u w}^{j l}}^{01}\right] \\
& +\frac{1}{2}\left[\left(\frac{a}{c}\right)^{2} C_{u}^{k l} I_{\phi_{u u}^{i k}}^{0000} J_{\psi_{u u}^{j l}}^{11}+\frac{a}{c} C_{w}^{k l} I_{\phi_{u w}^{i k}}^{0010} J_{\psi_{u w}^{j l}}^{10}\right. \\
& \left.\left.+\left(\frac{a}{b}\right)^{2} C_{u}^{k l} I_{\phi_{u u}^{i k}}^{0101} J_{\psi_{u u}^{j l}}^{00}+\frac{a}{b} C_{\mathrm{v}}^{k l} I_{\phi_{u \mathrm{v}}^{i k}}^{0110} J_{\psi_{u \mathrm{v}}}^{00}\right]\right\}, \quad, \quad \text { A }
\end{aligned}
$$$$
\frac{\partial U_{\max }}{\partial C_{\mathrm{v}}^{i j}}=\frac{b c E}{a(1+\nu)} \sum_{k=1}^{m} \sum_{l=1}^{n}\left\{\frac{1-\nu}{1-2 \nu}\left(\frac{a}{b}\right)^{2} C_{\mathrm{v}}^{k l} I_{\phi_{\mathrm{vv}}}^{0101} J_{\psi_{\mathrm{vv}}}^{00}\right.
$$$$
+\frac{\nu}{1-2 \nu}\left[\frac{a}{b} C_{u}^{k l} I_{\phi_{\mathrm{v} u}}^{0110} J_{\psi_{\mathrm{v} u}^{00}}^{00}+\frac{a^{2}}{b c} C_{w}^{k l} I_{\phi_{\mathrm{v} w}}^{0100} J_{\psi_{\mathrm{v} w}}^{01}\right]
$$$$
+\frac{1}{2}\left[\left(\frac{a}{c}\right)^{2} C_{\mathrm{v}}^{k l} I_{\phi_{\mathrm{vv}}^{i k}}^{0000} J_{\psi_{\mathrm{v}}}^{11}+\frac{a^{2}}{b c} C_{w}^{k l} I_{\phi_{\mathrm{v} w}^{i k}}^{0001} J_{\psi_{\mathrm{v}}}^{10}\right.
$$$$
\left.\left.+\frac{a}{b} C_{u}^{k l} I_{\phi_{\mathrm{v} u}^{i k}}^{1001} J_{\psi_{\mathrm{v} u}^{j l}}^{00}+C_{\mathrm{v}}^{k l} I_{\phi_{\mathrm{vv}}^{i k}}^{1010} J_{\psi_{\mathrm{vv}}^{j l}}^{00}\right]\right\},
$$$$
\frac{\partial U_{\max }}{\partial C_{w}^{i j}}=\frac{b c E}{a(1+\nu)} \sum_{k=1}^{m} \sum_{l=1}^{n}\left\{\frac{1-\nu}{1-2 \nu}\left(\frac{a}{c}\right)^{2} C_{w}^{k l} I_{\phi_{w w}^{0000} J_{\psi_{w w}^{j l}}^{11}}\right.
$$$$
+\frac{\nu}{1-2 \nu}\left[\frac{a^{2}}{b c} C_{\mathrm{v}}^{k l} I_{\phi_{w \mathrm{v}}}^{0001} J_{\psi_{w \mathrm{v}}^{j l}}^{10}+\frac{a}{c} C_{u}^{k l} I_{\phi_{w u}^{i k}}^{0010} J_{\psi_{w u}^{j l}}^{10}\right]
$$$$
+\frac{1}{2}\left[\frac{a^{2}}{b c} C_{v}^{k l} I_{\phi_{w v}^{i k}}^{0100} J_{\psi_{w v}^{j l}}^{01}+\left(\frac{a}{b}\right)^{2} C_{w}^{k l} I_{\phi_{w w}^{i k}}^{0101} J_{\psi_{w w}^{j l}}^{00}\right.
$$$$
\left.\left.+\frac{a}{c} C_{u}^{k l} I_{\phi_{w u}^{10}}^{1000} J_{\psi_{w u}^{01}}^{01}+C_{w}^{k l} I_{\phi_{w w}^{1 i}}^{1010} J_{\psi_{w w}^{j l}}^{00}\right]\right\},
$$$$
\frac{\partial T_{\max }}{\partial C_{u}^{i j}}=\rho \omega^{2} a b c \sum_{k=1}^{m} \sum_{l=1}^{n} C_{u}^{k l} I_{\phi_{u u}^{i k}}^{0000} J_{\psi_{u u}^{j l}}^{00},
$$$$
\frac{\partial T_{\max }}{\partial C_{\mathrm{v}}^{i j}}=\rho \omega^{2} a b c \sum_{k=1}^{m} \sum_{l=1}^{n} C_{\mathrm{v}}^{k l} I_{\phi_{\mathrm{v}}}^{0000} J_{\psi_{\mathrm{vv}}^{j l}}^{00},
$$$$
\frac{\partial T_{\max }}{\partial C_{w}^{i j}}=\rho \omega^{2} a b c \sum_{k=1}^{m} \sum_{l=1}^{n} C_{w}^{k l} I_{\psi_{w w}^{i k}}^{000} J_{\psi_{w w}^{j l}}^{00}
$$

where the integral notations $I_{\phi_{\alpha \beta}^{i k}}^{a b c d}$ and $J_{\psi_{\alpha \beta}^{j l}}^{e f}$ are given in Eqs. (19a), (19b).

\section{APPENDIX B}

The maximum strain energy in a vibration cycle for a parallelepiped neglecting $\sigma_{z z}$ is as follows:

$$
\begin{aligned}
U_{\max }= & \frac{b c E}{a(1+\nu)} \iiint_{V}\left\{\frac { 1 } { 2 ( 1 - \nu ) } \left[\left(\frac{\partial U}{\partial \bar{x}}\right)^{2}\right.\right. \\
& \left.+\left(\frac{a}{b}\right)^{2}\left(\frac{\partial V}{\partial \bar{y}}\right)^{2}\right]+\frac{a \nu}{b(1-\nu)} \frac{\partial U}{\partial \bar{x}} \frac{\partial V}{\partial \bar{y}} \\
& +\frac{1}{4}\left[\left(\frac{a}{c}\right)^{2}\left(\frac{\partial V}{\partial \bar{z}}\right)^{2}+\frac{2 a^{2}}{b c} \frac{\partial V}{\partial \bar{z}} \frac{\partial W}{\partial \bar{y}}+\left(\frac{a}{b}\right)^{2}\left(\frac{\partial W}{\partial \bar{y}}\right)^{2}\right. \\
& +\left(\frac{a}{c}\right)^{2}\left(\frac{\partial U}{\partial \bar{z}}\right)^{2}+\frac{2 a}{c} \frac{\partial U}{\partial \bar{z}} \frac{\partial W}{\partial \bar{x}}+\left(\frac{\partial W}{\partial \bar{x}}\right)^{2}+\left(\frac{a}{b}\right)^{2}\left(\frac{\partial U}{\partial \bar{y}}\right)^{2} \\
& \left.\left.+\frac{2 a}{b} \frac{\partial U}{\partial \bar{y}} \frac{\partial V}{\partial \bar{x}}+\left(\frac{\partial V}{\partial \bar{x}}\right)^{2}\right]\right\} d \bar{x} d \bar{y} d \bar{z} .
\end{aligned}
$$

\section{APPENDIX C}

The stiffness matrix element expressions for a parallelepiped neglecting $\sigma_{z z}$ are as follows:

$k_{u u}^{i k j l}=\frac{1}{1-\nu} I_{\phi_{u u}^{i k}}^{1010} J_{\psi_{u u}^{j l}}^{00}+\frac{1}{2}\left[\left(\frac{a}{c}\right)^{2} I_{\phi_{u u}^{0 i k}}^{0000} J_{\psi_{u u}^{j l}}^{11}+\left(\frac{a}{b}\right)^{2} I_{\phi_{u u}^{i k}}^{0101} J_{\psi_{u u}^{j l}}^{00}\right]$,

$k_{u \mathrm{~V}}^{i k j l}=\frac{\nu}{1-\nu} \frac{a}{b} I_{\phi_{u \mathrm{~V}}^{i k}}^{1001} J_{\psi_{u \mathrm{~V}}^{j l}}^{00}+\frac{a}{2 b} I_{\phi_{u \mathrm{~V}}^{i k}}^{010} J_{\psi_{u \mathrm{~V}}^{j l}}^{00}$,

$k_{u w}^{i k j l}=\frac{a}{2 c} I_{\phi_{u w}^{i k}}^{0010} J_{\psi_{u w}^{j l}}^{10}$,

$k_{\mathrm{vv}}^{i k j l}=\frac{1}{1-\nu}\left(\frac{a}{b}\right)^{2} I_{\phi_{\mathrm{vv}}^{i k 1}}^{0101} J_{\psi_{\mathrm{vv}}^{j l}}^{00}+\frac{1}{2}\left[\left(\frac{a}{c}\right)^{2} I_{\phi_{\mathrm{vv}}^{i k}}^{0000} J_{\psi_{\mathrm{vv}}^{j l}}^{11}+I_{\phi_{\mathrm{vv}}^{i k}}^{1010} J_{\psi_{\mathrm{vv}}^{j l}}^{00}\right]$,

$k_{\mathrm{v} w}^{i k j l}=\frac{a^{2}}{2 b c} I_{\phi_{\mathrm{v} w}^{i k}}^{0001} J_{\psi_{\mathrm{v} w}^{j l}}^{10}$,

$k_{w w}^{i k j l}=\frac{1}{2}\left[\left(\frac{a}{b}\right)^{2} I_{\phi_{w w}^{i k}}^{0101} J_{\psi_{w}^{j l}}^{00}+I_{\phi_{w w}^{1 i k}}^{1010} J_{w w}^{00}\right]$.

Bert, C. W., and Chen, T. L. C. (1978). "Effect of shear deformation on vibration of antisymmetric angle-ply laminated rectangular plates," Int. J. Solids Struct. 14, 465-473.

Fromme, J. A., and Leissa, A. W. (1970). "Free vibration of the rectangular parallelepiped,"' J. Acoust. Soc. Am. 48, 290-298.

Gould, P. L. (1988). Analysis of Shells and Plates (Springer, New York). p. 507.

Hutchinson, J. R. (1967). "Axisymmetric vibrations of a solid elastic cylinder encased in a rigid container," J. Acoust. Soc. Am. 42, 398-402.

Hutchinson, J. R. (1971). "Axisymmetric vibration of a free finite-length rod," J. Acoust. Soc. Am. 51, 223-240.

Hutchinson, J. R. (1979). "Axisymmetric flexural vibrations of a thick free circular plate," Trans. ASME, J. Appl. Mech. 46, 139-144.

Hutchinson, J. R. (1980). "Vibrations of solid cylinders," Trans. ASME, J. Appl. Mech. 47, 901-907.

Hutchinson, J. R. (1981). "Transverse vibration of beams: Exact versus approximate solutions," Trans. ASME, J. Appl. Mech. 48, 923-928.

Hutchinson, J. R. (1984). "Vibrations of thick free circular plates, exact versus approximate solutions," Trans. ASME, J. Appl. Mech. 51, 581585.

Hutchinson, J. R., and Zillmer, S. D. (1983). "Vibration of a free rectangular parallelepiped," Trans. ASME, J. Appl. Mech. 50, 123-130.

Iyengar, K. T. S., Chandrashekhara, K., and Sebastian, V. K. (1974). "'On the analysis of thick rectangular plates," Arch. Appl. Mech. 43, 317-330. 
Leissa, A. W. (1969). Vibration of Plates, NASA SP-160.

Leissa, A. W., and Zhang, Z. D. (1983). "On the three-dimensional vibrations of the cantilevered rectangular parallelepiped,' J. Acoust. Soc. Am. 73, 2013-2021.

Leissa, A. W., and So, J. (1995a). "Comparisons of vibration frequencies for rods and beams from one-dimensional and three-dimensional analyses,', J. Acoust. Soc. Am. 98, 2122-2135.

Leissa, A. W., and So, J. (1995b). "Accurate vibration frequencies of circular cylinders from three-dimensional analysis,' J. Acoust. Soc. Am. 98, 2136-2141.

Leissa, A. W., and So, J. (1995c). "Three-dimensional vibrations of truncated hollow cones,' J. Vibration Control 1, 145-158.

Levinson, M. (1980). "An accurate simple theory of the statics and dynamics of elastic plates," Mech. Res. Commun. 7, 343-350.

Liew, K. M., Hung, K. C., and Lim, M. K. (1995a). 'Free vibration studies on stress-free three-dimensional elastic solids,' Trans. ASME, J. Appl. Mech. 62(1), 159-165.

Liew, K. M., Hung, K. C., and Lim, M. K. (1995b). “'Vibration of stressfree hollow cylinders of arbitrary cross section,' 'Trans. ASME, J. Appl. Mech. 62(3), 718-724.

Liew, K. M., and Lim, C. W. (1996). “'A higher-order theory for vibration of doubly-curves shallow shells,' Trans. ASME, J. Appl. Mech. 63(3), $587-593$.

Lim, C. W., and Liew, K. M. (1995). "A higher order theory for vibration of shear deformable cylindrical shallow shells,', Int. J. Mech. Sci. 37(3), 277-295.

Lim, C. W., and Liew, K. M. (1996). "Vibration of moderately thick cylindrical shallow shells," J. Acoust. Soc. Am. 100, 3665-3673.

Lim, C. W., Liew, K. M., and Kitipornchai, S. (1998a). "Numerical aspects for free vibration of thick plates. Part I: Formulation and verification,", Comput. Methods Appl. Mech. Eng. 156(1-4), 15-29.

Lim, C. W., Kitipornchai, S., and Liew, K. M. (1998b). "Numerical aspects for free vibration of thick plates. Part II: Numerical efficiency and vibration frequencies.' Comput. Methods Appl. Mech. Eng. 156(1-4), 31-44.

Lim, C. W., Liew, K. M., and Kitipornchai, S. (1998c). "Vibration of open cylindrical shells: A three-dimensional elasticity approach,' J. Acoust. Soc. Am. (in press).
Lo, K. H., Christensen, R. M., and Wu, E. M. (1977a). “A higher-order theory of plate deformation, Part I: Homogeneous plates,' Trans. ASME, J. Appl. Mech. 44, 663-668.

Lo, K. H., Christensen, R. M., and Wu, E. M. (1977b). "A higher-order theory of plate deformation, Part 2: Laminated plates,' Trans. ASME, J. Appl. Mech. 44, 669-676.

Mindlin, R. D. (1951). "Influence of rotatory inertia and shear on flexural motions of isotropic, elastic plates," Trans. ASME, J. Appl. Mech. 18, 31-38.

Qatu, M. S. (1993). "Theories and analyses of thin and moderately thick laminated composite curves beams,', Int. J. Solids Struct. 30(20), 27432756.

Reddy, J. N. (1984a). Energy and Variational Methods in Applied Mechanics (Wiley, New York).

Reddy, J. N. (1984b). " A simple higher-order theory for laminated composite plates,' Trans. ASME, J. Appl. Mech. 51, 745-752.

Reddy, J. N., and Khdeir, A. A. (1989). "Buckling and vibration of laminated composite plates using various plate theory,', Amer. Inst. Aero Astro J. 27, 1808-1817.

Reddy, J. N., and Phan, N. D. (1985). "Stability and vibration of isotropic, orthotropic and laminated plates according to a higher-order shear deformation theory,', J. Sound Vib. 98, 157-170.

Reissner, E. (1945). "The effect of transverse shear deformation on the bending of elastic plates,' Trans. ASME, J. Appl. Mech. 12, A69-A77.

So, J., and Leissa, A. W. (1997). "Free vibrations of thick hollow circular cylinders from three-dimensional analysis,' Trans. ASME, J. Vib. Acoust. 119, 89-95.

Soler, A. (1968). "Higher order effects in thick rectangular elastic beams,", Int. J. Solids Struct. 4, 723-739.

Washizu, K. (1982). Variational Methods in Elasticity and Plasticity, 3rd ed. (Pergamon Oxford). pp. 276-279, 599-600.

Whitney, J. M., and Sun, C. T. (1973). "A higher order theory for extensional motion of laminated composites," J. Sound Vib. 30, 85-97.

Whitney, J. M., and Sun, C. T. (1974). "A refined theory for laminated, anisotropic cylindrical shells,' Trans. ASME, J. Appl. Mech. 41(2), 471476. 